\title{
Levetiracetam-Induced Psychosis in a Patient with Epilepsy
}

José Fernando Hernández $\mathbf{P}^{1^{*}}$, Julio César Moreno $\mathbf{C}^{1}$, Jeimy Natalia Giraldo Urrea ${ }^{2}$, Juan Pablo Durán $\mathbf{A}^{2}$ and Sergio $F$ Ramirez $\mathbf{G}^{1}$

${ }^{1}$ Children's Hospital, University San Jose, Bogotá, Colombia

'University Foundation of Health Sciences, Bogotá, Colombia

\begin{abstract}
Epilepsy is one of the most common diseases in the neurological practice. Its pharmacological treatment includes the use of antiepileptic drugs, which in general have a wide margin of tolerability and a good side effect profile. However, antiepileptic drug treatment can induce psychosis in some patients. Risk in increased with certain medications such as levetiracetam.
\end{abstract}

Keywords: Levetiracetam; Pharmacological treatment; Antiepileptic drugs; Psychosis; Epilepsy

\section{Introduction}

Epilepsy is one of the most common neurological disorders in the world and in our continent and one of the most frequent reasons for consultation in both the outpatient and emergency departments [1].

It affects near 50 million people worldwide, of this $80 \%$ are in developing countries. In Colombia, a prevalence of 10.1 per 1,000 people is estimated, with little variation between regions [2].

Levetiracetam, one of the second generation antiepileptic drugs, is known to cause behavioral side effects ranging from anxiety, agitation and less frequently psychotic episodes [3-5].

We report a case of 52-year-old female presenting in our hospital with a first interictal psychotic episode secondary to the use of levetiracetam.

\section{Case Presentation}

A 52-year-old female was admitted in our hospital, Hospital Universitario Infantil de San José, with a medical history of 5 months of visual and somatostatic hallucinations referred as "snakes and worms". On the day before admission, she suffers a generalized tonic-clonic seizure. There is not history of substance abuse.

She has a diagnoses of epilepsy since 12-year-old with focal seizure with impaired awareness and generalized tonic-clonic seizures initially treated with valproic acid and five months ago switched to levetiracetam $500 \mathrm{mg}$ bid as monoterapy.

At initial evaluation the patient was disoriented, perseverating with hallucinations referred as "I have snakes and worms on me" and she tried to take them off of her. The rest of the neurologic exam was no relevant.

During hospitalization patient presents an episode of agitation, irritability and persists with hallucination.

Diagnostic workup included a blood cell count, thyroid function test, urine analysis, computed tomography and magnetic resonance imaging of the brain which were all normal. EEG monitoring/video record was made for a 24 hours without ictal activity but it showed slow background with bilateral anterior asynchronous temporal interictal activity (Figures 1 and 2).

Based on history, physical examination and diagnostic test there was a high suspicion of a first episode of interictal psychosis induced by levetiracetam.

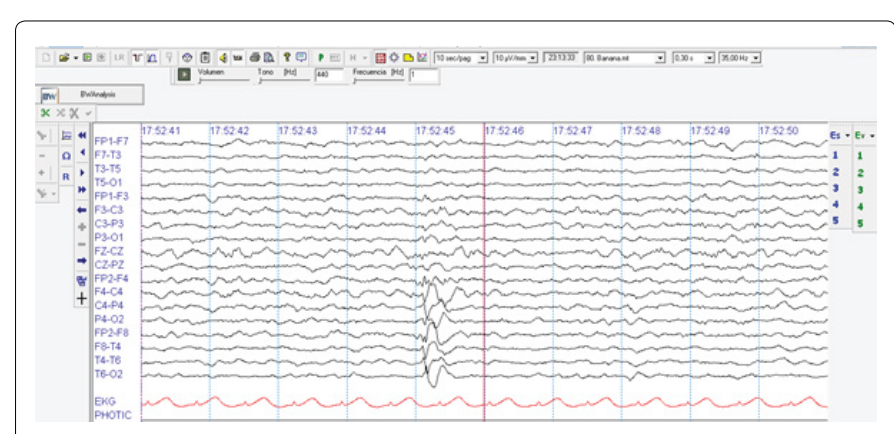

Figure 1: Spike slow wave complex maximal right anterior temporal (arrow).

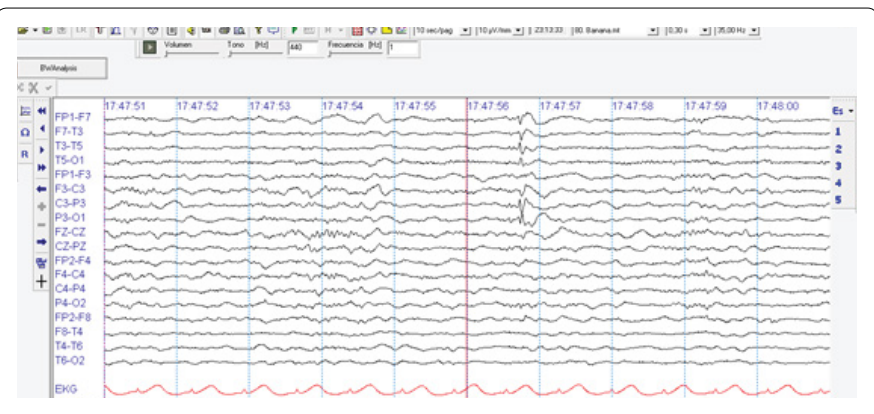

Figure 2: Spike slow wave complex maximal left anterior temporal (arrow).

Levetiracetam was stopped and we started valproic acid 500 mg every 12 hours and risperidone. The patient recovered from her psychotic symptoms over 48-72 hours.

\section{Discussion}

Patients with epilepsy are at increased risk and are more vulnerable to having psychiatric co-morbidity including psychosis which increases severity and disability secondary to pathology [6].

Psychosis of epilepsy refers to a group of psychotic disorders

*Corresponding author: José Fernando Hernández P, Children's Hospital University San Jose, Bogotá, Colombia, Tel: +5714377540; E-mail: josefernandoh@ gmail.com

Received: June 02, 2017; Accepted: July 24, 2017; Published: July 31, 2017

Citation: Hernández JFP, Moreno JCC, Urrea JNG, Durán JPA, Ramirez SFG (2017) Levetiracetam-Induced Psychosis in a Patient with Epilepsy. J Psychiatry 20: 422. doi:10.4172/2378-5756.1000422

Copyright: @ 2017 Hernández JFP, et al. This is an open-access article distributed under the terms of the Creative Commons Attribution License, which permits unrestricted use, distribution, and reproduction in any medium, provided the original author and source are credited 
with a distinct phenomenology in which potential etiopathogenic mechanisms are related to the epilepsy disorder [7]. Psychosis of epilepsy can result from seizure recurrence and remits with seizure control. This is the reason why this disorder should be evaluated and treated by neurologists and epileptologists.

Diagnosis of psychotic disorders require the presence of hallucinations (auditory, visual or tactile) and/or delusions (persecutory, referential, somatic, religious or grandiose) associated with a disorganized thinking and abnormal motor behavior (classification system DSM-5), these symptoms must last at least 1 day and it must be sufficiently severe to impaired patient's social or occupational function [6].

Psychosis of epilepsy can be classified in two categories: episodic psychosis of epilepsy and chronic or nonepisodic psychosis of epilepsy. The first category is linked to the status of seizure control. In the other hand, second category has not relation with seizure control, episodes can last from few days to several weeks and has a more guarded prognosis [7].

Other classifications separate the psychotic disorders according to their temporal relationship with seizure occurrence and the use or not $o$ antiepileptic drugs (AED) [7]. According to this, psychotic disorders can be divided in: antiepileptic drug-induced psychosis (ADIP), interictal psychosis of epilepsy, ictal psychosis and post-ictal psychosis $[6,7]$. ADIP represents an iatrogenic adverse drug reaction. Prevalence of ADIP has been reported between $1.0-8.4 \%$ and it is clear diagnosis just can be made retrospectively [6]. It requires that the psychotic symptoms developed during or soon after the exposure to an AED. Interictal psychosis of epilepsy is defined as psychotic episodes when they present independently of seizures. Ictal psychosis presents as an expression of seizure activity [7]. Post-ictal psychosis is defined as psychotic episodes when they occur within 7 days of a seizure or a cluster of seizures [6,7]. There is an alternative psychosis as a separate category in which the onset of the psychosis follows the suppression of the seizures [7].

Levetiracetam is the most common AED used among patients with ADIP either as monotherapy or in combination with other AEDs even at low doses of $500 \mathrm{mg}-1000 \mathrm{mg}$ every 12 hours [6]. It is in the group of the second generation AED, approved by the FDA en 1999 for the treatment of focal seizures or focal to bilateral seizures [4-9] initially combined with other AED but nowadays is used in monotherapy. It is a (-)-(S)- $\alpha$-metil-2-oxo-1-pyrrolidine acetamide [10]. Its mechanism of action is not well known, but it is thought to act by binding to synaptic vesicle protein $2 \mathrm{~A}$ (SV2A) modulating its actions affecting, finally, neural excitability [5-11].

Although levetiracetam has wide margin of tolerability and a good side effect profile with infrequent and mild adverse event over a dose range of 500 to $5000 \mathrm{mg}$ for adults or $20-30 \mathrm{mg} / \mathrm{kg}$ for children [4,11], behavioral disturbance has been noted in $2 \%$ of patients and depression prevalence in a multicenter study was $1.9 \%$ of patients taking $1000 \mathrm{mg} /$ day and $5.7 \%$ in patients taking $2000 \mathrm{mg}$ /day to 4\% [4]. Psychiatric adverse effects are seen in up to $13.3 \%$ of adults and $37.6 \%$ of pediatric patients [3-5]. Even thought psychotic symptoms are less frequent data about them are available only in case reports and they are more stressful and disabling for both the patient and their relatives.

\section{Conclusion}

Some studies has shown no relationship between the starting dose or titration of levetiracetam suggesting that susceptibility of patient is more important and relevant [4]. Other studies suggest that patients with epilepsy are biologically more vulnerable to psychosis of epilepsy [12].

In the present case, taking into account a temporal relationship between the onset of levetiracetam as monotherapy for the control of epilepsy and the presentation of psychotic symptoms, with the diagnostic workup within normality, findings of electroencephalography and substantial improvement after stop of levetiracetam, the possibility of a levetiracetam-induced psychosis is likely.

In the antiepileptic drugs induced psychosis the neurologists intervention is crucial to the prevention and remission of psychotic symptoms. It is very important to have a strict neurological and psychiatric surveillance in patients with levetiracetam.

\section{References}

1. Salud OPdl, Salud OMdl (2013) Informe sobre la Epilepsia en América Latina $y$ el Caribe.

2. Velez A, Eslava-Cobos J (2006) Epilepsy in Colombia: Epidemiologic profile and classification of epileptic seizures and syndromes. Epilepsia 47: 193-201.

3. Dannaram S, Borra D, Pulluri M, Jindal P, Sharma A (2012) Levetiracetaminduced acute psychotic episode. Innov Clin Neurosci 9: 10-12.

4. Mula M, Trimble MR, Yuen A, Liu RS, Sander JW (2003) Psychiatric adverse events during levetiracetam therapy. Neurology 61: 704-706.

5. Kumar N, Swaroop HS, Chakraborty A, Chandran S (2014) Levetiracetam induced acute reversible psychosis in a patient with uncontrolled seizures. Indian J Pharmacol 46: 560-561.

6. Chen Z, Lusicic A, O'Brien TJ, Velakoulis D, Adams SJ, et al. (2016) Psychotic disorders induced by antiepileptic drugs in people with epilepsy. Brain 139 2668-2678.

7. Kanner AM (2000) Psychosis of Epilepsy: A neurologist's perspective. Epilepsy Behav 1: 219-227.

8. Hung OS, Richard (2011) Antiepileptic drugs: The old and the new. Emerg Med Clin North Am 29: 141-150.

9. Fisher RS, Cross JH, D'Souza C, French JA, Haut SR, et al. (2017) Instruction manual for the ILAE 2017 operational classification of seizure types. Epilepsia 58: $531-542$

10. Goldenberg MM (2010) Overview of drugs used for epilepsy and seizures: Etiology, diagnosis, and treatment. PT 35: 392-415.

11. Verrotti A, Prezioso G, Di Sabatino F, Franco V, Chiarelli F, et al. (2015) The adverse event profile of levetiracetam: A meta-analysis on children and adults. Seizure 31: 49-55.

12. French J, Edrich P, Cramer JA (2001)A systematic review of the safety profile of levetiracetam: A new antiepileptic drug. Epilepsy Res 47: 77-90. 